\title{
Variable Asymmetry of the Circumstellar Envelope in IK Tauri Traced by SiO Maser Emission
}

\author{
Naoko Matsumoto*, ${ }^{*}$ Hiroshi Imai, ${ }^{b}$ Toshihiro Omodaka ${ }^{b}$ and VERA Project Team \\ ${ }^{a}$ Department of Astronomical Sciences, The Graduate University for Advanced Studies \\ Mitaka, Tokyo 181-8588, Japan \\ ${ }^{b}$ Faculty of Science, Kagoshima University \\ Kagoshima, Kagoshima 890-0065, Japan \\ E-mail: naoko.matsumoto@nao.ac.jp
}

We present the VLBI Exploration of Radio Astrometry (VERA) observations of $43 \mathrm{GHz}(J=$ $1-0, v=1$ and $v=2) \mathrm{SiO}$ maser emission toward the Mira variable IK Tauri, over nine times from November 2003 to April 2005. The SiO maser distributions were highly variable with time. The velocity structures were complex. The relative maser distributions between $v=1$ and $v=2$ show that $42-50 \%$ of the maser pairs are located within 0.5 mas. It suggests that the maser pumping is likely to be dominated by line overlapping or collisional pumping, rather than pure radiative pumping.

The 9th European VLBI Network Symposium on The role of VLBI in the Golden Age for Radio Astronomy and EVN Users Meeting September 23-26, 2008

Bologna, Italy

* Speaker. 


\section{Introduction}

IK Tauri (NML Tau, IRC+10050) is an oxygen-rich Mira variable exhibiting strong $\mathrm{SiO}$ maser emission in its circumstellar envelope. The pulsation period is about 470 days (Wing \& Lockwood 1973; Hale et al. 1997), and the distance is estimated between 250-270 pc (Olofsson et al. 1998; Whitelock et al. 1994). The map of $\mathrm{SiO}(J=1-0, v=1)$ maser emission around IK Tauri was obtained with VLBA once (Boboltz \& Diamond 2005). That map shows an elliptical asymmetric ring of the emission, extending to an area of approximately $58 \times 32 \mathrm{mas}^{2}$. Its major axis is directed northeast-southwest, and line-of-sight velocities of the masers show systematic differences; blueshifted and red-shifted masers are located in the northwest and southeast, respectively. Matsumoto et al. (2008) performed the VLBI observations of the $\mathrm{SiO}(J=1-0, v=1$ and $v=2)$ maser transitions toward IK Tauri for nine-epochs that covers one pulsation period to investigate the variation of maser structure on the time scale of 510 days.

\section{Observations}

$\mathrm{SiO}(J=1-0, v=1$ and $v=2)$ masers $43 \mathrm{GHz}$ in IK Tauri have been observed using VERA over nine times from Nov. 2003 to May 2005. Although VERA is dedicated for maser astrometry using the dual-beam system, only one beam was used for observations presented here, because of the absence of a bright position reference calibrator near IK Tauri. In the present observations, therefore, the accurate absolute coordinates of IK Tauri cannot be known. The synthesized beam was less than 0.9 mas $\times 0.6$ mas.

\section{Results and discussion}

Figure 1 shows the totally integrated maps of all velocity channels. Here, these maps show the spots that have a signal-to-noise ratio larger than 7 in the velocity channel maps. The $\mathrm{SiO}$ maser spots around IK Tauri typically show elliptical or quadrilateral distributions. The position angles of the elongation directions were changed with time from the northwest-southeast direction to northeast-southwest direction. They are found in right side and left side of figure 1, respectively. The velocity structures were complex, and it does not provide any direct evidence for a rotational SiO maser shell proposed by Boboltz \& Diamond (2005).

Figure 2 shows the number distribution of the angular distances from each $v=1$ spot to the nearest $v=2$ spot having a radial velocity within $\sim 0.22 \mathrm{~km} \mathrm{~s}^{-1}(= \pm 1 \mathrm{ch})$ of that of the $v=1$ spot. This is the same as with figure 6 in Matsumoto et al. (2008). From figure 2, 42\% of the maser pair of the $v=1$ and $v=2$ spots have an angular distance of less than 0.5 mas at 28 Feb. 2004. It shows that the overall distributions of the $v=1$ and $v=2$ maser spots are similar to each other. This trend favors a scenario in which line overlapping and/or collisional pumping are dominat, rather than the pure radiative pumping mechanism. In the future, 2-beam phase referencing observations with VERA and precise astrometry better than 1 mas would be helpful for better understanding the SiO pumping mechanism. In addition to VERA 2-beam observations (e.g., Kamohara et al. 2008), pumping models that treat the degree of spot coincidence between the $v=1$ and $v=2 \mathrm{SiO}$ maser lines will be required for further understanding this issue. 

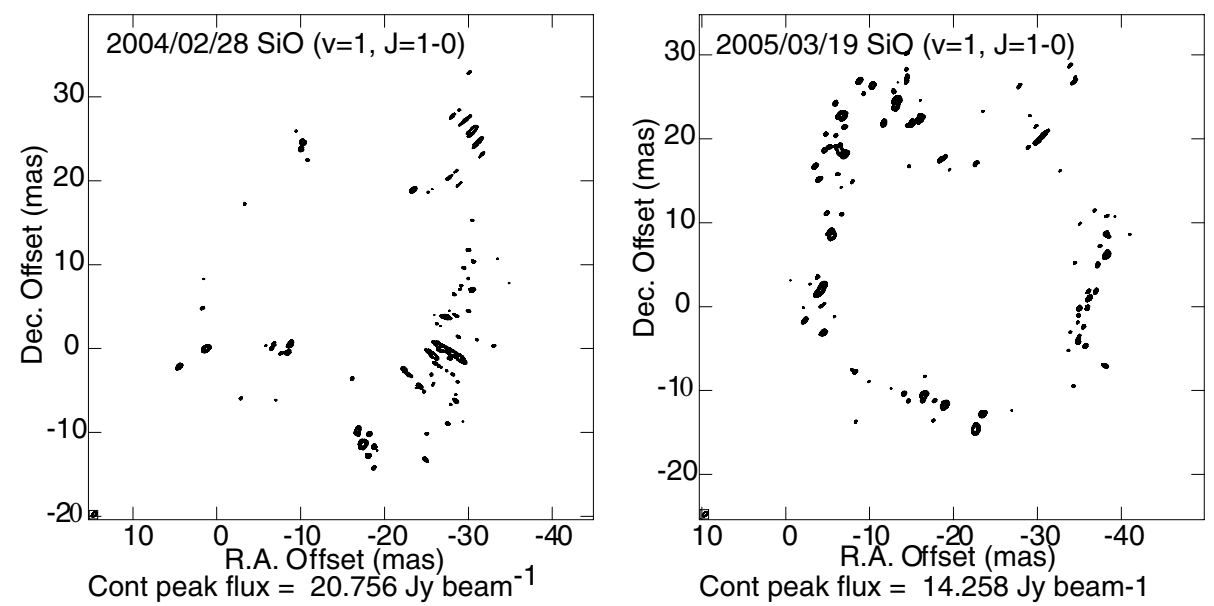

Figure 1: Intensity maps of the $\mathrm{SiO}(J=1-0, v=1)$ masers towards IK Tauri, obtained by integration of all velocity channels.
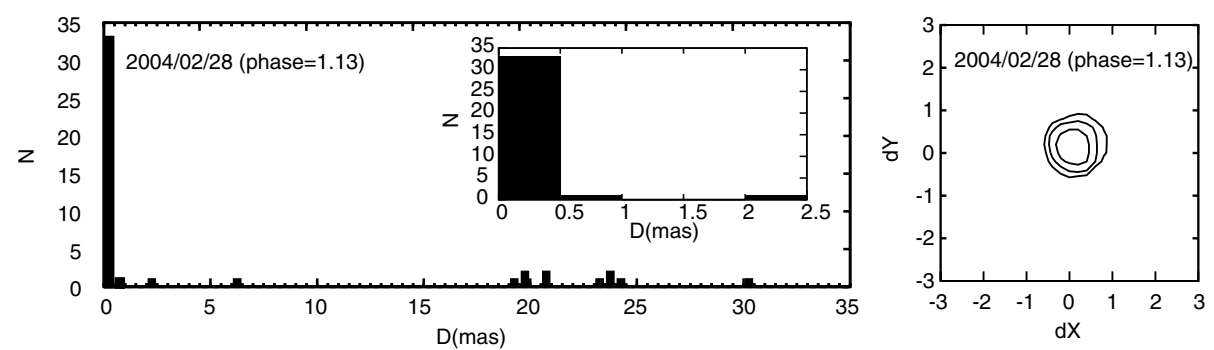

Figure 2: Left: Number distribution of the angular distances from each $v=1$ spot to the nearest $v=2$ spot at nearly same velocity channel $( \pm 1 \mathrm{ch})$. Zoom-in view around a small $D$ value is inserted. Right: The number distribution of $v=1$ and $v=2$ maser pairs with the angular distance less 0.5 mas, with varying relative offsets in $X$ and $Y$ coordinates between $v=1$ and $v=2$ maps. One contour level corresponds to 10 pairs of $v=1$ and $v=2$ masers.

\section{References}

[1] Boboltz, D. A., \& Diamond, P. J. 2005, ApJ, 625, 978

[2] Hale, D. D. S., et al. 1997, ApJ, 490, 407

[3] Kamohara, R, et al. 2008, PASJ, 60, 1023

[4] Olofsson, H., Lindqvist, M., Nyman, L. - $\AA$.,\& Winnberg, A. 1998, A\&A, 329, 1059

[5] Matsumoto, N., et al. 2008, PASJ, 60, 1039

[6] Whitelock, P., Menzies, J., Feast, M., Marang, F., Carter, B., Roberts, G., Catchpole, R., \& Chapman, J. 1994, MNRAS, 267, 711

[7] Wing, R. F.,\& Lockwood, G. W. 1973, ApJ, 184, 873 